\title{
A New Construction For Invariant Numerical Schemes Using Moving Frames
}

\author{
Marx Chhay ${ }^{\mathrm{a}}$, Aziz Hamdouni ${ }^{\mathrm{a}}$ \\ ${ }^{\mathrm{a}}$ LEPTIAB - Université de La Rochelle, Avenue Michel Crépeau, 17042 La Rochelle Cedex 01, FRANCE \\ Received *****; accepted after revision +++++ \\ Presented by
}

\begin{abstract}
We propose a new approach for moving frame construction that allows to make finite difference scheme invariant. This approach takes into account the order of accuracy and guarantees numerical properties of invariant schemes that overcome those of classical schemes. Benefits obtained with this process are illustrated with the Burgers equation.

To cite this article: A. Chhay, A. Hamdouni, C. R. Mecanique 333 (2009).
\end{abstract}

\section{Résumé}

Une construction nouvelle des schémas invariants utilisant les repères mobiles. On propose une procédure nouvelle de construction des repères mobiles permettant de rendre invariant les schémas de discrétisation en différences finies. Elle prend en compte l'ordre de consistance et garantit aux schémas invariants de meilleures performances que celles des schémas classiques. On illustre les performances de cette approche sur l'exemple de l'équation de Burgers.

Pour citer cet article: M. Chhay, A. Hamdouni, C. R. Mecanique 333 (2009).

Key words: Invariant scheme; Lie symmetry ; Moving frame; Burgers ; Geometric integrator ;

Mots-clés : Schéma invariant; Symétrie de Lie; Repères mobiles; Burgers ; Intégrateur géométrique

\section{Version française abrégée}

Les méthodes numériques construites afin de préserver certaines propriétés liées à la structure géométrique des équations s'appelle les intégrateurs géométriques. Elles permettent de traduire naturellement le comportement qualitatif des solutions ainsi que de réduire les instabilités numériques. En particulier, les

Email addresses: nchhay01@univ-lr.fr (Marx Chhay), aziz.hamdouni@univ-lr.fr (Aziz Hamdouni). 
schémas invariants permettent de conserver le groupe de symétrie des équations et de réduire les erreurs numériques. Une méthode de construction de tels schémas a été développée par M. Fels et P. J. Olver. Elle est basée sur le concept de repère mobile. Dans cette procédure, la qualité des solutions numériques d'un schéma invariant est entièrement conditionnée par le choix du repère mobile associé au groupe de symétrie. Ce choix est déterminé par le procédé de normalisation d'É. Cartan qui permet de ramener la détermination du répère mobile associé à un groupe continue au choix d'une section transverse de l'orbite d'un élément. Ce procédé possède l'avantage d'exhiber une famille importante de schémas invariant mais ne garantit pas au schéma obtenu des qualités numériques meilleures que le schéma d'origine. Nous proposons une méthode de construction nouvelle des schémas invariants utilisant les repères mobiles. Cette méthode peut être décrite sous la forme algorithmique suivante : (i) On considère un schéma discrétisant une EDP et le groupe de symétrie dépendant de paramètres réels de l'EDP, (ii) on transforme le schéma afin d'obtenir un schéma paramétrisé, (iii) on suppose une forme algébrique des paramètres en fonction de coefficients réels, (iv) on calcule les conditions d'équivariance afin que les paramètres de transformation deviennent des repères mobiles, (v) enfin, on calcule les conditions sur les coefficients réels pour que le schéma transformé soit d'un ordre de précision fixé. Ainsi on obtient un schéma invariant dont l'ordre de consistance est déterminé. On illustre les performances de cette approche á travers la construction d'un schéma invariant pour l'équation de Burgers.

\section{Introduction}

As a direct legacy of E. Galois ${ }^{1}$ and thanks to the works of S. $\mathrm{Lie}^{2}$, Lie group methods provide powerfull tools for differential equations analysis [3][6]. From the symmetry group of equations, precious informations of the behaviour of the solutions can be deduced, as the conservation laws of the system or the existence of selfsimilar solutions. The applications are various problems in mechanics, mathematical physics, and in fluid dynamics [1][2][4]. On the numerical side, Lie group integrators are known to be successful in the reliable reproduction of qualitative behaviours in a solution and also in reducing numerical instabilities. A recent approach to construct invariant numerical schemes using moving frames, as developed by M. Fels and P. J. Olver, allows to preserve symmetry of equations and reduce numerical errors. The method has been studied and validated for many classes of differential systems and for partial differential equations (PDE) by P. Kim [5]. The numerical properties of such invariant schemes are completely dependent on the choice of the moving frame. This one is determined by geometric arguments following the Cartan 's method of normalization. This process has the great advantage to propose a large familly of invariantized schemes, but it also suffers dramatically due to the possibility of computing averaged invariant schemes whose properties can be worser than initial classical schemes. In this paper, the authors propose an alternate way of constructing invariant numerical schemes that guarantees the order of accuracy.

\section{Principle of construction}

\subsection{Definitions}

In this subsection some definitions needed for the construction of invariant numerical schemes are briefly recalled. For more details, we refer the reader to the works of P. Kim [5], P. J. Olver [7]. Consider a PDE

\footnotetext{
1 Evariste Galois, french mathematician, 1811-1832

2 Sophus Lie, norwegian mathematician, 1842-1899
} 
$F(z)=0$ over a manifold $M$. A symmetry of $F(z)=0$ is a group of transformations $G$ that preserves the set of whole solutions of the equation :

$$
F(z)=0 \Rightarrow F(g \cdot z)=0 \quad \forall g \in G
$$

A numerical application $N$ that stays unchanged under any element of $G$ is said to be $G$-invariant :

$$
N(z)=N(g \cdot z) \quad \forall g \in G
$$

For the present purpose, numerical schemes are considered as numerical applications that verify some consistency conditions with the continuous PDE [5]. A numerical scheme is therefore $G$-invariant if its transformation coincides with itself. Let's now look at particular elements $\rho(z)$ of the group $G$ that depends on the variable $z$ and that verify the equivariance relation :

$$
\rho(g \cdot z)=\rho(z) g^{-1} \quad \forall g \in G
$$

Such transformations are called moving frames associated to the group $G$. The fundamental theorem of construction of invariant numerical schemes using moving frames simply states that if a numerical scheme is transformed by a moving frame associated to the group $G$, then the scheme becomes $G$-invariant [5]. The new approach proposed in this paper allows to fix the order of accuracy of the invariant scheme. It can be summarize as follows : start with a classical numerical scheme associated to a PDE whose symmetry group noted $G$ has $d$ finite dimensional 1-parameter subgroups, then (i) express the $G$-transformed scheme in function of the symmetrization parameters $\varepsilon_{k}$, for $k=1, \ldots, d$, (ii) for each $\varepsilon_{k}, k=1, \ldots, d$, suppose an algebraic form involving the stencil points of the original scheme and some real constant coefficients $\alpha_{k}^{1}, \ldots, \alpha_{k}^{\sigma}$, where $\sigma$ is the number of stencil points. (iii) Compute the value of the real constant coefficients such that : a) each $\varepsilon_{k}, k=1, \ldots, d$, verifies the equivariance property (equ. 3 ), and b) the parametrized scheme is at less as accurate than the original scheme. One finally obtains an invariant scheme with a chosen order of accuracy.

Let's compute by this process an invariant explicit numerical scheme for the Burgers' equation. Numerical results are illustrated in the next section.

\subsection{Symmetry of the Burgers equation}

Consider the Burgers equation for the dependent variable $u$ in a spatial domain $\Omega$ for time $t \geq 0$ :

$$
\frac{\partial u}{\partial t}+u \frac{\partial u}{\partial x}=\nu \frac{\partial^{2} u}{\partial x^{2}}
$$

associated with the initial condition $u(x, 0)=u_{0}(x)$, and the condition $u_{\left.\right|_{\partial \Omega}}=g(t)$ on the boundary $\partial \Omega$ of the domain. The parameter $\nu$ is the viscosity. The symmetry group of (4) is composed by the one-parameter transformations [6] : spatial translation : $(x, t, u) \longmapsto\left(x+\varepsilon_{1}, t, u\right)$; time translation : $(x, t, u) \longmapsto\left(x, t+\varepsilon_{2}, u\right)$; projection : $(x, t, u) \longmapsto\left(\frac{x}{1-\varepsilon_{3} t}, \frac{t}{1-\varepsilon_{3} t},\left(1-\varepsilon_{3} t\right) u+\varepsilon_{3} x\right)$; scale transformation : $(x, t, u) \longmapsto\left(x e^{\varepsilon_{4}}, t e^{2 \varepsilon_{4}}, u e^{-\varepsilon_{4}}\right) ;$ galilean boost $:(x, t, u) \longmapsto\left(x+\varepsilon_{5} t, t, u+\varepsilon_{5}\right)$.

For scheme invariance considerations, the interest is focused on all symmetries except the scale transformation. Indeed, most of numerical schemes are invariant under the space translation, the time translation and the scale transformation. The projection and the galilean boost are most often broken. Consider the transformation $(x, t, u) \longmapsto(\bar{x}, \bar{t}, \bar{u})$ depending on parameters $\varepsilon_{1}, \varepsilon_{2}, \varepsilon_{3}$ and $\varepsilon_{5}$ :

$$
\bar{x}=\frac{\left(x+\varepsilon_{1}\right)+\varepsilon_{5}\left(t+\varepsilon_{2}\right)}{1-\varepsilon_{3}\left(t+\varepsilon_{2}\right)}, \quad \bar{t}=\frac{t+\varepsilon_{2}}{1-\varepsilon_{3}\left(t+\varepsilon_{2}\right)}, \quad \bar{u}=u\left(1-\varepsilon_{3}\left(t+\varepsilon_{2}\right)\right)+\left(x+\varepsilon_{1}\right) \varepsilon_{3}+\varepsilon_{5},
$$


Let's make invariant under this transformation the method defined by the classical explicit Forward in Time and Centered in Space scheme (FTCS scheme) :

$$
\frac{u_{j}^{n+1}-u_{j}^{n}}{\Delta t}+u_{j}^{n}\left(\frac{u_{j+1}^{n}-u_{j-1}^{n}}{2 \Delta x}\right)-\nu \frac{u_{j+1}^{n}-2 u_{j}^{n}+u_{j-1}^{n}}{\Delta x^{2}}=0
$$

This scheme is computed on a regular and orthogonal mesh such that $\Delta t=t^{n+1}-t^{n}$ and $\Delta x=x_{j+1}-x_{j}$. The discretisation stencil is composed by the discrete points $\mathbf{z}=\left(z_{j}^{n+1}, z_{j}^{n}, z_{j \pm 1}^{n}\right)$, where $z_{j}^{n}=\left(x_{j}, t^{n}, u_{j}^{n}\right)$. For the transformation acting only on the independent variables (the time and the space translations), the same argument as in [5] is used. In order to express the numerical scheme in term of the time step $\Delta t$ and the spatial step $\Delta x$, choose for moving frames :

$$
\varepsilon_{1}=-x_{j}, \quad \varepsilon_{2}=-t^{n},
$$

So that we now consider the action of the group $G$ over the stencil points $\mathbf{z}$ :

$$
\begin{aligned}
z_{j}^{n} & \mapsto \bar{z}_{j}^{n}=\left(0,0, u_{j}^{n}+\varepsilon_{5}\right) \\
z_{j}^{n+1} & \mapsto \bar{z}_{j}^{n+1}=\left(\frac{\varepsilon_{5} \Delta t}{1-\varepsilon_{3} \Delta t}, \frac{\Delta t}{1-\varepsilon_{3} \Delta t}, u_{j}^{n+1}\left(1-\varepsilon_{3} \Delta t\right)+\varepsilon_{5}\right) \\
z_{j \pm 1}^{n} & \mapsto \bar{z}_{j \pm 1}^{n}=\left(\Delta x, 0, u_{j \pm 1}^{n}+\varepsilon_{3} \Delta x+\varepsilon_{5}\right)
\end{aligned}
$$

In order to make $\varepsilon_{3}$ and $\varepsilon_{5}$ the moving frames and then to make $\rho$ a moving frame associated to the transformation (8) of $G$, and to compute the equivariance relation (3), let's choose for their expression a combination of discrete variables which are dimensionally relevant (as $\left[\varepsilon_{3}\right]=\left[\right.$ time $\left.^{-1}\right) \cdot \varepsilon_{3}$ must not have any term in $u^{n+1}$ to keep the explicit form of the numerical scheme. The symmetrization parameter must be at most of degree one to preserve the order of the convective term. Finally the construction of a moving frame from the appplication $\varepsilon_{3}$ requires that there are no terms depending on $\Delta x$ and $\Delta t$ alone. Similar arguments for $\varepsilon_{5}$ (of dimension [length] $\times[\text { time }]^{-1}$ ) can be built. Suppose then the algebraic form :

$$
\varepsilon_{3}=\frac{a u_{j+1}^{n}+b u_{j}^{n}+c u_{j-1}^{n}}{\Delta x}, \quad \varepsilon_{5}=d u_{j+1}^{n}+e u_{j}^{n}+f u_{j-1}^{n},
$$

where $a, b, c$ and $d, e, f$ are constant real coefficients. Then write down the expression of any transformed stencil points $\overline{\mathbf{z}}=g \cdot \mathbf{z}$ for $g \in G$ by $\rho$ taking into account the expression of $\varepsilon_{3}$ and $\varepsilon_{5}$ given in (11). Substitute then the expression of $\overline{\mathbf{z}}$ following the action (8) of $G$, the equivariance condition (3) gives the restriction over the coefficients :

$$
c-a=1, \quad a+b+c=0, \quad d-f=0, \quad d+e+f=-1,
$$

The invariant FTCS (IFTCS) scheme is then :

$$
\frac{u_{j}^{n+1}-u_{j}^{n}}{\Delta t}+u_{j}^{n}\left(\frac{u_{j+1}^{n}-u_{j-1}^{n}}{2 \Delta x}\right)-\nu \frac{u_{j+1}^{n}-2 u_{j}^{n}+u_{j-1}^{n}}{\Delta x^{2}}+F=0
$$

with the constant coefficients $(a, b, c, d, e, f)$ verifying (12) in :

$$
\begin{aligned}
F= & -2\left(\frac{a u_{j+1}^{n}+b u_{j}^{n}+c u_{j-1}^{n}}{\Delta x}\right)\left(u_{j}^{n+1}-u_{j}^{n}\right)+\Delta t\left(\frac{a u_{j+1}^{n}+b u_{j}^{n}+c u_{j-1}^{n}}{\Delta x}\right)^{2} u_{j}^{n+1} \\
& +\left(d u_{j+1}^{n}+e u_{j}^{n}+f u_{j-1}^{n}\right)\left(\frac{u_{j+1}^{n}-u_{j-1}^{n}}{2 \Delta x}+\left(\frac{a u_{j+1}^{n}+b u_{j}^{n}+c u_{j-1}^{n}}{\Delta x}\right)\right)
\end{aligned}
$$

The order of accuracy of the IFTCS scheme is obtained by Taylor expansion. The added term is consistent with : 


$$
\begin{aligned}
F= & (a+c) \frac{\Delta x}{2} \frac{\partial^{2} u}{\partial x^{2}}\left(-u-2 \Delta t \frac{\partial u}{\partial t}\right) \\
& +\Delta t\left(2 \frac{\partial u}{\partial x} \frac{\partial u}{\partial t}+\left(u+\Delta t \frac{\partial u}{\partial t}\right)\left(-\frac{\partial u}{\partial x}+(a+c) \frac{\Delta x}{2} \frac{\partial^{2} u}{\partial x^{2}}\right)^{2}\right)+O\left(\Delta t, \Delta x^{2}\right)
\end{aligned}
$$

The condition for the transformed scheme to be of the same order of accuracy as the original classical FTCS scheme is then $a+c=0$. Therefore $\varepsilon_{3}$ has an unique expression $\varepsilon_{3}=-\left(u_{j+1}^{n}-u_{j-1}^{n}\right)(2 h)^{-1}$. The expression of $\varepsilon_{5}$ is given by (11). The coefficients $d, e, f$ verify the equivariance condition (12).

\section{Numercial results}

\subsection{Pseudo-shock test}

Consider the Burgers equation (4) on a bounded domain $\Omega$. The initial and the boundary conditions are given by the following exact solution :

$$
\left.u_{\text {exact }}(x, t)=\frac{-\sinh \left(\frac{x}{2 \nu}\right)}{\cosh \left(\frac{x}{2 \nu}\right)+\exp \left(-\frac{t}{4 \nu}\right)}, \quad \quad \quad \Omega=\right]-1,1[, \quad t \geq 0,
$$

When the viscosity is very small $\nu \rightarrow 0$, a shock appears. Figure (1) illustrates the behaviour of the numerical solution associated to the implicit Crank-Nicolson scheme (CN scheme) and the explicit invariant FTCS scheme (IFTCS scheme), in comparison to the analytical solution (16). The sizes of the grid are set to $\Delta x=\Delta t=5 \cdot 10^{-2}$. The viscosity $\nu$ is fixed at $75 \cdot 10^{-5}$ (it corresponds to an instability region for the classical FTCS scheme : its solution is completely blown up).

The solution of the CN scheme presents non physical oscillations around high gradient zone. In opposition, the numerical solution of the IFTCS scheme stays close to the analytical solution. Wriggles are avoided but a slight dissipation appears.

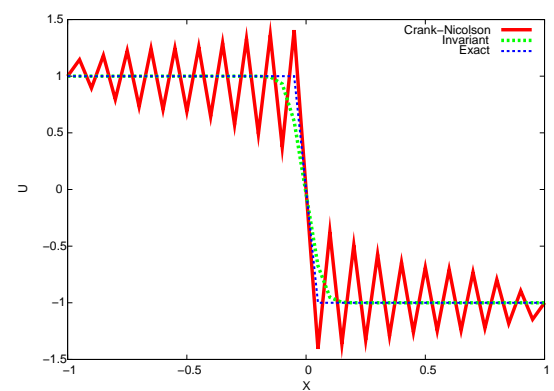

FIG. 1. Burgers equation. Exact solution, implicit Crank-Nicolson scheme, and explicit invariant scheme. Over the spatial domain $\Omega=]-1 ; 1\left[\right.$ at time $t=2$. Mesh parameters are set to $\Delta x=\Delta t=5.10^{-2}$, and the viscosity is $\nu=75.10^{-5}$.

\subsection{Galilean invariance.}

As the set of whole solutions of Burgers equation is invariant under galilean tansformation. The authors test how the numerical solution depends on the referential frame from which it is observed. It is done by the application of a galilean boost $\lambda$ to the original referential frame $\Omega=]-2 ; 2[$. Figure (2) shows 
the numerical behaviour of (a) : the classical FTCS scheme, and (b) : the IFTCS scheme. Space size step is set to $\Delta x=2.10^{-2}$. Time step size is computed in order to keep the CFL number constant to $1 / 2$. Viscosity is fixed at $\nu=5.10^{-3}$. We observe that the solution of the FTCS becomes rapidly degraded as $\lambda$ grows. The solution blows up when $\lambda \geq 1$. It is no more the case when the scheme inherits the property of invariance. The solution remains unaffected irrespective of the value of $\lambda$.

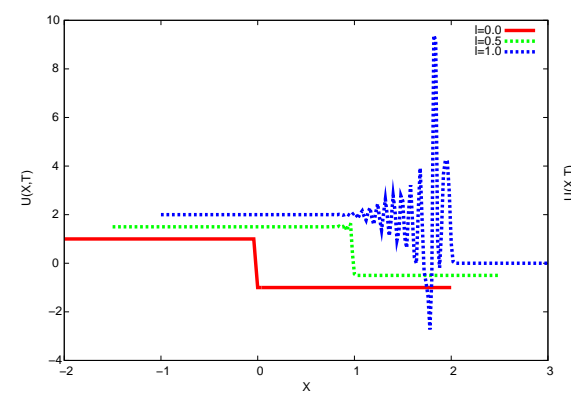

(a) FTCS scheme

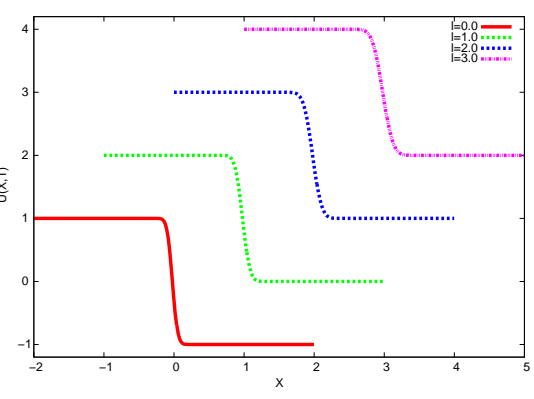

(b) Invariant FTCS scheme

FIG. 2. Burgers equation. Behaviour of numerical solution of (a) : the FTCS scheme, (b) : the IFTCS scheme, for different galilean boost $\lambda$. Space step size $\Delta x$ is $2.10^{-2}$. Viscosity $\nu$ is $5.10^{-3}$. CLF is conserved at $1 / 2$. Time $t=2$.

\section{Conclusion}

The authors propose a new approach for construction of invariant numerical schemes using moving frames. This approach is quite different from those proposed by Kim [5], in the sense that the proposed approach is based on numerical rather than geometrical considerations. The advantage of the present method is that it assures good numerical properties in addition to the preservation of the symmetry group of the continuous equation. The application of this method to the explicit FTCS scheme for the Burgers equation yields a stable solution with good accuracy. Invariance has also allowed to respect the principle of galilean independance which is broken by the classical scheme.

\section{Références}

[1] B. J. Cantwell. "Introduction to Symmetry Analysis". Cambridge Univ. Press, Cambridge, 2002.

[2] P. E. Hydon. "Symmetry Methods for Differential Equations". Cambridge Univ. Press, Cambridge, 2000.

[3] N. H. Ibragimov. "CRC Handbook of Lie Group Analysis of Differential Equations, vol. 1, Symmetry, Exact Solutions and Conservation Laws". CRC Press, Boca Raton, 1994.

[4] N. H. Ibragimov. "CRC Handbook of Lie Group Analysis of Differential Equations, vol. 2, Applications in Engineering and Physical Sciences". CRC Press, Boca Raton, 1995.

[5] P. Kim. "Invariantization of the Crank Nicolson Method For Burgers Equation". Physica D : Nonlinear Phenomena, 237 (2) (2008), 243-254.

[6] P. J. Olver. "Applications of Lie Groups To Differential Equations". Springer-Verlag. (2nd), 1993.

[7] P. J. Olver, "Moving Frames". J. Symb. Comp. 3 (2003), 501-512. 
REPORT (12 jun 2009)

The note deals with an interesting subject, but it is written in terrible english. There are errors of grammar, of style, and confusions between words and phrases that pile up in each sentence.

It is not possible to avoid misunderstanding the sentences in these condition. The authors should thoroughly rewrite their paper.

This article has been corrected following the report instructions 
REPORT (2 sep 2009)

In the paper, a new approach to construct invariant numerical schemes for Burgurs equations is presented. The authors adopt the moving frame method and suggest a different criterion to create moving frames. There are two major points that I suggest for improvement of the paper.

1. Main difference between the authors' work and that of P. Kim lies in choice of moving frames. Their approach seems to put more emphasis on a numerical aspect, rather than geometrical one. However, the strategy for choice of moving frame adopted in the paper is not clearly described. I believe it should be explained in a general context first and then applied to specific equations. This is necessary, especially when considering the paper's title.

2. The background information necessary to understand the authors' work is not properly presented in the paper. For examples, many researchers in applied mathematics are not familiar with the concept of symmetries in differential equations, let alone the moving frame. The authors excessively omit the details, simply referring to some references. This makes most readers, even in the numerical analysis community, difficult to understand their work.

I suggest that the paper may be accepted after proper modifications are made for the points raised above.

1. As the expert has noted, the presented new algorithm to construct moving frames (and then to compute invariant schemes with a choosen order of accuracy) has been described in the particular case of the Burgers equation. The way it has been written lets underlied the general process, but after this second correction, the general case has been described in an algorithmic way at the end of subsection 2.1. :

It can be summarize as follows : start with a classical numerical scheme associated to a PDE whose symmetry group noted $G$ has d finite dimensional 1-parameter subgroups, then (i) express the G-transformed scheme in function of the symmetrization parameters $\varepsilon_{k}$, for $k=1, \ldots, d$, (ii) for each $\varepsilon_{k}, k=1, \ldots, d$, suppose an algebraic form involving the stencil points of the original scheme and some real constant coefficients $\alpha_{k}^{1}, \ldots, \alpha_{k}^{\sigma}$, where $\sigma$ is the number of stencil points. (iii) Compute the value of the real constant coefficients such that : a) each $\varepsilon_{k}, k=1, \ldots, d$, verifies the equivariance property (equ. 3), and b) the parametrized scheme is at less as accurate than the original scheme. One finally obtains an invariant scheme with a chosen order of accuracy. 
2. The constrain of six pages needed for a submission to the "Comptes Rendus de l'Académie des Sciences" implies some necessarily references to the scientific background to understand the purpose. An explicit recall of all notions can really not be possible. The introduction of the main concepts of modern differential geometry (and their formalism) used here would have taken probably more than six pages (specially when the basic features do not belong to the common background of researchers in applied mathematics or computational mechanics, as underlined by the expert). In the present case, only short motivations of the geometric approach and some classical references on the subject can be cited in introduction of the paper :

As a direct legacy of Évariste Galois (french mathematician, 1811-1832) and thanks to the works of Sophus Lie (norwegian mathematician, 1842-1899), Lie group methods provide powerfull tools for differential equations analysis [3][6]. From the symmetry group of equations, precious informations of the behaviour of the solutions can be deduced, as the conservation laws of the system or the existence of selfsimilar solutions. The applications are various problems in mechanics, mathematical physics, and in fluid dynamics [1][2][4]. On the numerical side, Lie group integrators are known to be successful in the reliable reproduction of qualitative behaviours in a solution and also in reducing numerical instabilities.

The MOdifications BRought AND THE CORRECTIONS ADDED TO THE PAPER HAVE TAKEN INTO ACCOUNT THE REPORT OF THE EXPERT. 